\title{
ERRATUM
}

O. Stalnov • I. Fono • A. Seifert

\section{Erratum to: Closed-loop bluff-body wake stabilization via fluidic excitation}

(C) Springer-Verlag 2011

\section{Erratum to: Theor. Comput. Fluid Dyn. DOI 10.1007/s00162-010-0197-3}

In the paper by Stalnov et al. there has been a duplication of Fig. 5a such that it also appears as Fig. 4b. The correct Fig. 4b is shown below with its correct caption.

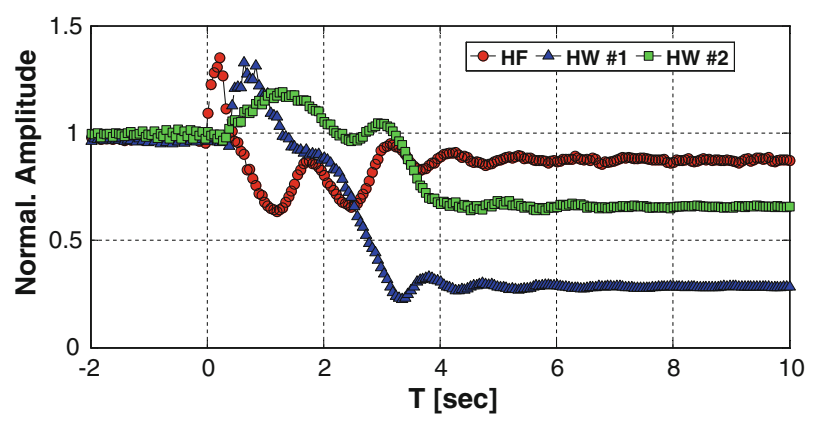

Fig. 4b Ensemble averaged (64 realizations) short-time standard deviation ( 256 time points, at a sampling rate of $\mathrm{Fs}=2.5 \mathrm{kHz}$ ) of two hot-wires (HW) located in the wake (spanwise distance between the two wires is approximately 5D) and the body-mounted hot-film (HF) shear sensor. At time $T=0$ the actuators were turned ON. The input signal to the actuators is amplitude modulation (AM) of a modulation signal from the VCO (at the natural vortex shedding frequency), and a carrier signal of $1.35 \mathrm{kHz}$. The closed-loop was locked to the hot-film (HF) body-mounted sensor. The amplitudes are normalized by their baseline uncontrolled magnitude

The online version of the original article can be forund under doi:10.1007/s00162-010-0197-3.

O. Stalnov $\cdot$ I. Fono $\cdot$ A. Seifert $(\varangle)$

School of Mechanical Engineering, Tel-Aviv University, Tel-Aviv, Israel

E-mail: seifert@eng.tau.ac.il 